\title{
Toward the Standardization of Mitochondrial Proteomics: The Italian Mitochondrial Human Proteome Project Initiative
}

Tiziana Alberio, ${ }^{\dagger, \Phi}$ Luisa Pieroni, ${ }^{\ddagger}, \Phi$ Maurizio Ronci, ${ }^{\ddagger},, \Phi$ Cristina Banfi, ${ }^{\perp}$ Italia Bongarzone, ${ }^{q}$ Patrizia Bottoni, ${ }^{\#}$ Maura Brioschi, ${ }^{\perp}$ Marianna Caterino, $\|, \square$ Clizia Chinello, ${ }^{\bullet}$ Antonella Cormio, $\triangle$ Flora Cozzolino, $\square, \ominus$ Vincenzo Cunsolo, ${ }^{\square}$ Simona Fontana, ${ }^{\square}$ Barbara Garavaglia, ${ }^{\bigcirc}$ Laura Giusti, $^{\varnothing}$ Viviana Greco, Antonio Lucacchini, ${ }^{\varnothing}$ Elisa Maffioli, Fulvio Magni, ${ }^{\bullet}$ Francesca Monteleone, Maria Monti, $\square$ Valentina Monti, ${ }^{\bigcirc}$ Clara Musicco, Giuseppe Petrosillo,,${ }^{\nabla}$ Vito Porcelli, $\triangle$ Rosaria Saletti, Roberto Scatena, ${ }^{\prime}$ Alessio Soggiu, Gabriella Tedeschi, ${ }^{, \Delta}$ Mara Zilocchi, ${ }^{\dagger}$ Paola Roncada, ${ }^{\odot}$ Andrea Urbani, ${ }^{*}, \#$ and Mauro Fasano ${ }^{*} \dagger$

${ }^{\dagger}$ Department of Science and High Technology, Università degli Studi dell’Insubria, Busto Arsizio I-21052, Italy

${ }^{\ddagger}$ IRCCS-Santa Lucia Foundation, Rome I-00143, Italy

${ }^{\S}$ Department of Medical, Oral, and Biotechnological Sciences, University G. d'Annunzio of Chieti-Pescara, Chieti I-66013, Italy

${ }^{\perp}$ Centro Cardiologico Monzino, IRCCS, Milan I-20138, Italy

${ }^{\text {II } D e p a r t m e n t ~ o f ~ E x p e r i m e n t a l ~ O n c o l o g y ~ a n d ~ M o l e c u l a r ~ M e d i c i n e, ~ F o n d a z i o n e ~ I R C C S ~ I s t i t u t o ~ N a z i o n a l e ~ d e i ~ T u m o r i, ~ M i l a n ~ I-20133, ~ I t a l y ~}$

\#Institute of Biochemistry and Clinical Biochemistry, School of Medicine - Catholic University, Rome I-00168, Italy

"Dipartimento di Medicina Molecolare e Biotecnologie Mediche, Università degli Studi di Napoli “Federico II", Naples I-80131, Italy

$\square$ CEINGE Biotecnologie Avanzate s.c.a.r.l., Naples I-80145, Italy

-Department of Medicine and Surgery, University of Milano-Bicocca, Monza I-20900, Italy

$\triangle$ Dipartimento di Bioscienze, Biotecnologie e Biofarmaceutica, Università di Bari, Bari I-70125, Italy

๑Dipartimento di Scienze Chimiche, Università degli Studi di Napoli "Federico II", Naples I-80126, Italy

Department of Chemical Sciences, University of Catania, Catania I-95125, Italy

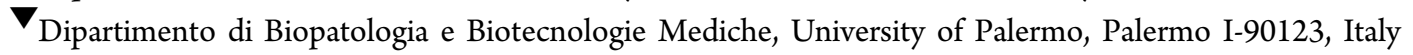

${ }^{\circ}$ Molecular Neurogenetics Unit, IRCCS Foundation Neurological Institute C. Besta, Milan I-20126, Italy

${ }^{\varnothing}$ Department of Pharmacy, University of Pisa, Pisa I-56126, Italy

Department of Veterinary Medicine (DiMeVet), University of Milan, Milan I-20133, Italy

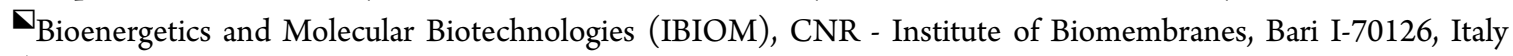

$\boldsymbol{\Delta}_{\text {Fondazione Filarete, Milan I-20139, Italy }}$

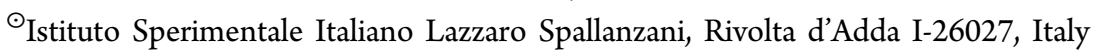

Supporting Information

ABSTRACT: The Mitochondrial Human Proteome Project aims at understanding the function of the mitochondrial proteome and its crosstalk with the proteome of other organelles. Being able to choose a suitable and validated enrichment protocol of functional mitochondria, based on the specific needs of the downstream proteomics analysis, would greatly help the researchers in the field. Mitochondrial fractions from ten model cell lines were prepared using three enrichment protocols and analyzed on seven different LC-MS/MS platforms. All data were processed using neXtProt as reference database. The data are available for the Human Proteome Project purposes through the ProteomeXchange Consortium with the identifier PXD007053. The processed data sets were analyzed using a suite of

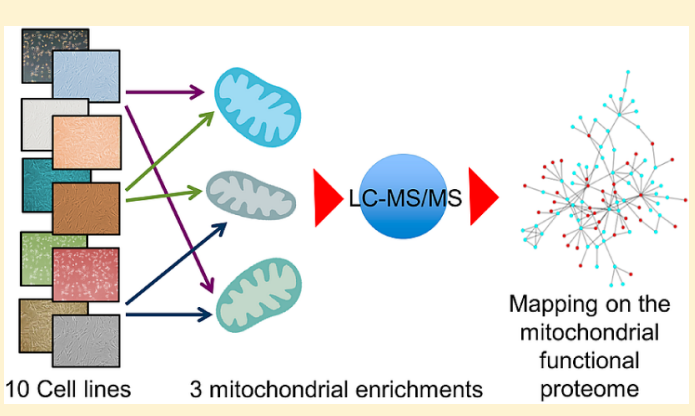
$\mathrm{R}$ routines to perform a statistical analysis and to retrieve subcellular and submitochondrial localizations. Although the overall Continued...

Special Issue: Chromosome-Centric Human Proteome Project 2017

Received: May 30, 2017

Published: August 22, 2017 
number of identified total and mitochondrial proteins was not significantly dependent on the enrichment protocol, specific line to line differences were observed. Moreover, the protein lists were mapped to a network representing the functional mitochondrial proteome, encompassing mitochondrial proteins and their first interactors. More than $80 \%$ of the identified proteins resulted in nodes of this network but with a different ability in coisolating mitochondria-associated structures for each enrichment protocol/cell line pair.

KEYWORDS: $\quad$ mitochondria, standardization, enrichment protocol, Mitochondrial Human Proteome Project

\section{INTRODUCTION}

The Mitochondrial Human Proteome Project (mt-HPP) is a Human Proteome Organization (HUPO) initiative, led by the Italian Proteomics Association (ItPA), focused on human mitochondrial proteins. ${ }^{1}$ Because of the intrinsic nature of the mitochondrial proteome, this action is part of both the Chromosomecentric Human Proteome Project (C-HPP) and the Biology/ Disease Human Proteome Project (B/D-HPP). The main goal of this effort is to obtain robust information about the integrative role of proteins acting at the mitochondrial level, considering both those encoded by the mitochondrial DNA (mt-DNA) and by the nuclear DNA.

Mitochondria are very versatile organelles, and they are involved in a plethora of cellular functions such as energy production, regulation of death pathways, and calcium buffering. ${ }^{2}$ Therefore, their dysfunction has been related to many pathological conditions ranging from neurodegenerative disorders to cancer and metabolic diseases. ${ }^{3-5}$ Hence, their dynamic proteome is particularly important in defining their healthy state, the dynamics of the mitochondrial network, and, in turn, the cellular conditions. ${ }^{6-8}$ The detection of proteins associated with mitochondria in specific physiological or pathological conditions is the main goal of several research groups and proteomics is the most effective approach to achieve this outcome. Nevertheless, the enrichment of mitochondria in the sample is needed to increase protein identification and quantification. The lack of reproducibility of many enrichment protocols, that leads to the introduction of uncontrolled bias in the manipulated specimen, is well-known and clearly detrimental for any subsequent analysis. It is not rare to find mitochondrial proteomics data reporting a low number of Matrix proteins. This is mainly due to the mitochondrial breakage along the enrichment procedure, with consequent leakage of matrix-mitochondrial proteins into the cytosol and the subsequent formation of membrane-surrounded artifacts, containing proteins originally not present in the organelle. In some cases, proteins associated with mitochondria, which define their interaction with the endoplasmic reticulum or with the autophagosome, are the most interesting ones. It is therefore fundamental to define the best protocol capable of preserving those interactions. Conversely, a different protocol could be best suited for studies requiring a deeper purification of the samples. It is well-known that the composition of mitochondrial proteome is tissue related and, hence, the best protocol depends on both the selected cellular model and the desired yield/purity.

For the above-mentioned reasons, a standardization effort aimed at defining the most appropriate extraction method for each cellular model in relation to a specific biological question is necessary. The Italian consortium working on this project includes 17 groups from 10 Universities and eight Research and Care Centers. Ten cell lines representative of different organs and tissues were selected (BJ, NCI-H28, Hek293, HeLa, HepG2, HUVEC, MDA-MB-231, THP1, U2OS, SH-SY5Y). They are all standard cell lines widely used for basic research; they are deeply characterized and, most importantly, are included in the panel used for the mapping of Cell Atlas subcellular locations within the Human Protein Atlas project. ${ }^{9}$ Some of them are also suitable cell models for particular pathological conditions related to the impairment of mitochondrial function.

Mitochondria were isolated with three different methods (differential centrifugation, sucrose gradient separation, a commercial kit based on surfactants). To collect homogeneous data, the samples prepared with different methods from the same cell line were analyzed by the same mass spectrometry (MS) laboratory. The yield and the purity of each enrichment were assessed by Western blot, before MS analysis. Moreover, integrity of some mitochondrial preparations was checked by measuring the oxygen consumption rate and the citrate synthase activity. Data elaboration with the commercial software PEAKS Studio 7.5 was centralized in one laboratory. Data sets were analyzed by an automated procedure, providing the coverage of subcellular and submitochondrial locations. Eventually, the identified proteins were mapped on a mitochondrial functional proteome network ${ }^{10}$ to verify the proportion of proteins associated with mitochondria.

\section{EXPERIMENTAL PROCEDURES}

\section{Cell Lines}

All the cell lines used (BJ, SH-SY5Y, U2OS, MDA-MB-231, NCI-H28, Hek293, HUVEC, THP1, HepG2, HeLa) are of human origin. Neuroblastoma SH-SY5Y (ECACC 94030304; Lot No. 11C016), osteosarcoma U2OS (ATCC HTB-96), skin fibroblast BJ (ATCC CRL-2522), embryo kidney Hek293 (ATCC CRL-1573), cervix adenocarcinoma Hela (ATCC CCL-2), hepatocellular carcinoma HepG2 (ATCC HB-8065), breast cancer derived MDA-MB-231 (ATCC HTB-26), normal umbilical vein HUVEC (ATCC PCS-100-010), lung metastasis derived NCI-H28 (ATCC CRL-5820), and acute monocytic leukemia THP1 (ECACC 88081201, Lot No. 14J009) cells were maintained at $37^{\circ} \mathrm{C}$ under humidified conditions and $5 \% \mathrm{CO}_{2}$ and were grown in the appropriate medium, as detailed in Table S-1. Cell culture media and other reagents were from Euroclone, Sigma, Corning, and Gibco Life Technologies. Absence of mycoplasma was periodically checked by EZ-PCR Mycoplasma Test Kit (Biological Industries) according to manufacturer's instructions.

\section{Mitochondrial Enrichment}

Mitochondria were isolated from $10^{7}-10^{8}$ cells, depending on the cell type. The number of cells and the lysis buffer volume used for each cell type are reported in Table S-1.

Differential Centrifugation. Cells were detached (if adherent) with trypsin $(0.05 \%)$-EDTA $(0.02 \%)$, collected by centrifugation $\left(300 \times g, 25^{\circ} \mathrm{C}, 7 \mathrm{~min}\right)$, and then resuspended in isolation buffer (250 mM sucrose, $10 \mathrm{mM}$ Tris/MOPS pH 7.4, $1 \mathrm{mM}$ EGTA, 10\% v/v protease inhibitor mix, P8340 Sigma-Aldrich). Cells were mechanically lysed with a glass/glass Dounce homogenizer, on ice, using a number of strokes optimized for each cell line. Homogenates were centrifuged $\left(600 \times g, 4{ }^{\circ} \mathrm{C}, 10 \mathrm{~min}\right)$ and the supernatants collected and further centrifuged $(7000 \times g$, $4{ }^{\circ} \mathrm{C}, 10 \mathrm{~min}$ ) to isolate the mitochondrial fractions. 
Sucrose Gradient Separation. Cells were washed with $1 \mathrm{mM}$ EDTA in PBS and, if adherent, detached with Trypsin (0.05\%)-EDTA (0.02\%). Cells were collected by centrifugation $\left(600 \times g, 25^{\circ} \mathrm{C}, 10 \mathrm{~min}\right)$ and then resuspended in mitochondrial buffer (MB: $210 \mathrm{mM}$ D-mannitol, $70 \mathrm{mM}$ sucrose, $1 \mathrm{mM}$ EDTA, $10 \mathrm{mM}$ Hepes, $\mathrm{pH} 7.5$ plus 1X Protease Inhibitor Cocktail, P8340 Sigma-Aldrich). Lysis was performed manually in a Teflon/ glass homogenizer in ice. Homogenization was repeated three times, each time with a number of strokes optimized for different cell lines (Hek293, $3 \times 30$ strokes; U2OS, $1 \times 50,2 \times 40$; HeLa, $3 \times 30$; SH-SY5Y, $3 \times 50$; NCI-H28, $1 \times 30,2 \times 20$; MDA-MB231, $3 \times$ 40; THP-1, $3 \times 50$; 1, $3 \times 30$ HUVEC). Homogenates were centrifuged $\left(600 \times g, 4{ }^{\circ} \mathrm{C}, 10 \mathrm{~min}\right)$ and supernatants collected, pooled together, and further centrifuged $\left(7000 \times g\right.$, at $\left.4{ }^{\circ} \mathrm{C}, 10 \mathrm{~min}\right)$ to collect the crude mitochondria fractions. The crude mitochondria pellets were suspended in $250 \mu \mathrm{L}$ of $\mathrm{MB}$ and layered on a discontinuous sucrose gradient (1.6 M/1.2 M sucrose in $10 \mathrm{mM}$ Hepes, $1 \mathrm{mM}$ EDTA, $0.1 \% \mathrm{BSA}$, at $\mathrm{pH} 7.5)$. The sucrose gradient was ultracentrifuged $(96000 \times g$, $4{ }^{\circ} \mathrm{C}, 2 \mathrm{~h}$ ) with acceleration and deceleration set to 3 , in a Beckman Ultracentrifuge equipped with a SW40Ti rotor in ultraclear tube (Beckman). Upon ultracentrifugation, visible as a whitish band at the gradient interface, mitochondria were collected with an insulin syringe, washed by dilution in four volumes of $\mathrm{MB}$, and collected by centrifugation $\left(13000 \times g, 4{ }^{\circ} \mathrm{C}, 10 \mathrm{~min}\right)$.

Commercial Kit Based on Surfactants. Cells were detached with trypsin (0.05\%)-EDTA (0.02\%) (if adherent) and collected by centrifugation. Mitochondria were isolated using the Mitochondrial Isolation Kit MITOISO2 (Sigma-Aldrich), following the detergent lysis protocol of the manufacturer. Briefly, after two washes in PBS, cells were lysed in lysis buffer with protease inhibitor cocktail (P8340 Sigma-Aldrich). Cells were then incubated for $5 \mathrm{~min}$ on ice before adding two volumes of extraction buffer and centrifuging $\left(600 \times g, 4{ }^{\circ} \mathrm{C}, 10 \mathrm{~min}\right)$. The supernatant was transferred in a fresh tube and centrifuged $\left(11000 \times g, 4{ }^{\circ} \mathrm{C}, 10 \mathrm{~min}\right)$ to obtain a pellet containing the mitochondria. The pellet was further washed with the extraction buffer to remove contaminants. For BJ and MDA-MB-231 cells only, the supernatant obtained after the first centrifugation was discarded to proceed to a second lysis of the cellular pellet with lysis buffer. A second centrifugation $\left(600 \times g, 4{ }^{\circ} \mathrm{C}\right.$, $10 \mathrm{~min}$ ) was performed after adding two volumes of extraction buffer.

After mitochondria enrichment, dry pellets from three independent preparations for each condition were stored at $-80{ }^{\circ} \mathrm{C}$ before MS and Western Blot analysis or directly used to measure the oxygen consumption rate.

\section{Western Blot}

Proteins were extracted from cells or subcellular-enriched pellets using radioimmunoprecipitation assay (RIPA) buffer $(50 \mathrm{mM}$ Tris- $\mathrm{HCl} \mathrm{pH} 7.6,150 \mathrm{mM} \mathrm{NaCl}, 1 \%$ sodium deoxycholate, $1 \%$ $\mathrm{NP}-40$ and $0.1 \% \mathrm{SDS})$. Lysates were centrifuged $(15000 \times \mathrm{g}$, $\left.4{ }^{\circ} \mathrm{C}, 30 \mathrm{~min}\right)$, and the supernatant was collected. Equal amount of proteins were incubated in Laemmli loading buffer, resolved in $10 \%$ or $13 \%$ SDS-gels and transferred to polyvinylidenedifluoride (PVDF) membranes (Millipore Corporation, Bedford, MA, USA) at $1.0 \mathrm{~mA} / \mathrm{cm}^{2}, 1.5 \mathrm{~h}$. PVDF membranes were saturated in tris-buffered saline with $0.05 \%$ Tween 20 (TBS-T) containing $5 \%$ dried skim milk. Blots were probed with antibodies against a nuclear marker (Histone H3, Sigma-Aldrich H0164, 1:2500), an outer mitochondrial membrane marker (Voltage-dependent anion channel 1 - VDAC1, Abcam ab15895, 1:1000), an inner mitochondrial membrane marker (cytochrome c oxidase subunit 5B - COX5 $\beta$, Sigma-Aldrich HPA034517, 1:1000), a mitochondrial matrix marker (citrate synthase - CS, Sigma-Aldrich AMAb91006, 1:1000), and a cytosolic marker ( $\beta$-tubulin, Thermo Scientific, MA5-16308, 1:8000). Blots were incubated with proper peroxidase-conjugated secondary antibodies, that is, antirabbit (Millipore Corporation AP132P, 1:1500) and antimouse (Millipore Corporation 12-349, 1:3000) in 5\% milk-TBS-T. Enhanced chemiluminescence substrate (Millipore Corporation) was used to visualize the peroxidase signals. Images ( 16 bit grayscale) were acquired using the G:BOXChemi XT4 (Syngene, Cambridge, UK) system.

\section{Mitochondrial Proteins Extraction and in-Solution Digestion}

To perform shotgun MS analysis of mitochondrial proteins, all the samples were lysed and digested in RapiGest Solution (RG, Waters Corporation): each mitochondrial pellet was resuspended in $50 \mu \mathrm{L}$ of RG $0.1 \%$ in $50 \mathrm{mM}$ ammonium bicarbonate and incubated on ice for $30 \mathrm{~min}$. Protein content was quantified using the Bradford protein assay (BIO-RAD). Before the digestion, proteins were reduced with $1 \mathrm{mM}$ TCEP, at $55{ }^{\circ} \mathrm{C}$ for $30 \mathrm{~min}$ and alkylated in the presence of $20 \mathrm{mM}$ iodoacetamide for $30 \mathrm{~min}$ in the dark, at RT.

Porcine Trypsin (Promega) was added at 50:1 protein to enzyme ratio and digestion ran overnight at $37^{\circ} \mathrm{C}$. Acidification with $0.5 \%$ TFA blocked the digestion reaction and, upon incubation at $37{ }^{\circ} \mathrm{C}$ for $30 \mathrm{~min}$, led to RG hydrolysis. The peptides were recovered by centrifugation at $15000 \times g$ for $10 \mathrm{~min}$ at $4{ }^{\circ} \mathrm{C}$ and then loaded directly on the respective chromatographic system for the MS analysis.

Liquid Chromatography and Mass Spectrometry Analysis (LC-MS/MS)

Mitochondrial peptides were analyzed using several MS platforms and chromatographic conditions (see Table 1) running a technical

\section{Table 1. MS and Chromatographic Platforms}

\begin{tabular}{|c|c|c|c|c|}
\hline no. & LC system & column & $\begin{array}{c}\text { mass } \\
\text { spectrometer }\end{array}$ & cells \\
\hline 1 & $\begin{array}{l}\text { nanoAcquity M } \\
\text { Class }\end{array}$ & $\begin{array}{l}\text { Waters HSST } 3 \mathrm{C}_{18} 75 \mu \mathrm{m} \times \\
15 \mathrm{~cm}\end{array}$ & Synapt G2 Si & BJ, HeLa \\
\hline 2 & $\begin{array}{r}\text { Dionex Ulti- } \\
\text { Mate } 3000\end{array}$ & 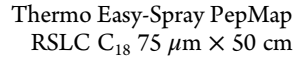 & $\begin{array}{l}\text { Orbitrap Fu- } \\
\text { sion }\end{array}$ & Hek293 \\
\hline 3 & $\begin{array}{r}\text { Dionex Ulti- } \\
\text { Mate } 3000\end{array}$ & $\begin{array}{l}\text { MS Wil GmbH C } 1875 \mu \mathrm{m} \times \\
20 \mathrm{~cm}\end{array}$ & $\begin{array}{l}\text { LTQ-Orbi- } \\
\text { trap- Velos }\end{array}$ & $\begin{array}{l}\text { MDA- } \\
\text { MB231, } \\
\text { THP1 }\end{array}$ \\
\hline 4 & nanoEASY II & $\begin{array}{l}\text { Nanoseparations } \mathrm{C}_{18} 100 \mu \mathrm{m} \\
\quad \times 20 \mathrm{~cm}\end{array}$ & $\begin{array}{l}\text { LTQ-Orbi- } \\
\text { trap-XL }\end{array}$ & NCI-H28 \\
\hline 5 & $\begin{array}{r}\text { Dionex Ulti- } \\
\text { Mate } 3000\end{array}$ & $\begin{array}{l}\text { Thermo Easy-Spray PepMap } \\
\text { RSLC } C_{18} 75 \mu \mathrm{m} \times 50 \mathrm{~cm}\end{array}$ & $\begin{array}{l}\text { Bruker Im- } \\
\text { pact } \mathrm{HD}\end{array}$ & HUVEC \\
\hline 6 & $\begin{array}{l}\text { Ekspert } \\
\text { nanoLC } 400\end{array}$ & $\begin{array}{l}\text { Thermo Acclaim PepMap } \\
10075 \mu \mathrm{m} \times 25 \mathrm{~cm}\end{array}$ & $\begin{array}{c}\text { TripleTOF } \\
5600+\end{array}$ & HepG2 \\
\hline 7 & nanoEASY II & $\begin{array}{l}\text { Thermo Acclaim PepMap } \\
10075 \mu \mathrm{m} \times 25 \mathrm{~cm}\end{array}$ & $\begin{array}{l}\text { Bruker maXis } \\
\text { HD }\end{array}$ & $\begin{array}{l}\text { SH-SY5Y, } \\
\text { U2OS }\end{array}$ \\
\hline
\end{tabular}

duplicate per sample. Details on all the instrumental setup used for the acquisition are provided as Supporting Information.

Data Analysis

Raw data were processed using PEAKS Studio 7.5 (Bioinformatics Solutions Inc., Waterloo, ON Canada) and searched against neXtProt (July 2017; 42151 total entries). Parent and Fragments Mass Error Tolerance were set according to the instrument or acquisition type and ranged from $10-40 \mathrm{ppm}$ for precursors and from $0.05-0.6 \mathrm{Da}$ for fragments. Other search parameters were trypsin enzyme specificity, two missed cleavages 
per peptide, one nonspecific cleavage, fixed carbamidomethylation of cysteines and variable oxidation of methionines, deamidation of glutamine and asparagine (NQ), with two variable PTM per peptide. FDR threshold on PSMs was set to $0.1 \%$, typically resulting in FDR on peptides and proteins lower than $0.2 \%$. In view of concatenating numerous data sets from several biological samples, very stringent FDR filtering conditions were chosen.

Protein identification tables for each biological replicate were analyzed using R (version 3.3.2), ${ }^{11}$ using the package UniProt.ws ${ }^{12}$ in the Bioconductor program. ${ }^{13}$ The complete code used is enclosed in the Supporting Information. Briefly, 63 tables (three biological replicates, two enrichment methods for nine cell lines, three enrichment methods for SH-SY5Y cells) in comma separated values format were read, and UniProtKB accession numbers and gene symbols were extracted. Proteins observed in a single biological replicate were discarded. Proteins were ranked by retaining all protein identifications whose computed score (-10Logp/ MW) was higher than a threshold value equal to $5.0 \times 10^{-4}$ with respect to the cumulative score ${ }^{14}$ (i.e., the sum of all scores for a sample). The number of filtered identifications was exported for each replicate. Gene symbols were then searched in MitoCarta2. $0^{15}$ and Integrated Mitochondrial Protein Index (IMPI Q2 2016) ${ }^{16}$ databases to obtain the number of entries annotated as mitochondrial in each biological replicate. Primary subcellular location category was also extracted from UniProt annotation (release 2017_07) to identify principal sources of contamination. Identities annotated as mitochondrial as the primary subcellular location were further classified as OMM (outer mitochondrial membrane), IMS (intermembrane space), IMM (inner mitochondrial membrane), Matrix, or NC (not classified). The same proportions were calculated in the neXtProt database. The Fisher's exact test was performed to compare results.

To evaluate if the number of identifications for the same cell line with different mitochondrial enrichment procedures was significantly different, a multiple $t$ test was performed on the number of total and mitochondrial identified proteins in each biological replicate. To compare the results of the three enrichment methods used for the SH-SY5Y cell line, one-way ANOVA was performed. The Tukey's posthoc test was used to identify significant multiple comparisons. In both cases, a $p$ value $<0.05$ was considered significant. Differences in the percentage of protein identities in the four most represented primary subcellular location categories were evaluated by the nonparametric Kruskal-Wallis test followed by the posthoc Dunn test.

Network Analysis

Consensus lists were mapped on the reference network for the functional mitochondrial proteome, ${ }^{10}$ a network composed of 6592 nodes ( 1361 of them labeled as "mitochondrial" in the neXtProt database and 5231 "mitochondrial associated" interactors in the IMEX consortium) and 16350 edges. Mapped nodes were extracted and visualized to obtain the number of "mitochondrial" and "mitochondrial associated" proteins for each cell line and enrichment method. The analysis was performed using Cytoscape (v3.4.0). ${ }^{17}$

\section{RESULTS}

\section{Samples Characterization Previous to MS Analysis}

To verify the enrichment yield and to confirm low levels of cytosolic and nuclear contaminations, all the specimens were first assessed by Western blots. A representative Western blot of the differently enriched mitochondrial proteins from SH-SY5Y cells is reported in Figure 1. Beta-tubulin was used as cytosolic marker, citrate synthase (CS) as mitochondrial Matrix marker, VDAC1 as OMM marker, cytochrome c oxidase $5 \mathrm{~B}(\mathrm{COX} 5 \beta)$ as IMM marker, and Histone H3 (H3) as nuclear marker. Figure 1 shows that mitochondrial fractions $(M)$ are enriched in markers from the three mitochondria compartment (Matrix, OMM, and IMM), cytosolic contamination is very low with respect to the total fraction $(T)$ and nuclear contamination is acceptable, if $\mathrm{H} 3$ levels are compared with those of the nuclear fraction $(N)$. Comparable enrichment results were obtained for the three methods. Western blots of preparations from all the other cell lines are reported in Figure S-1. Mitochondria from selected cell lines were also functionally tested in terms of integrity (citrate synthase assay) and respiration (oximetry or microscale oxygraphy), as shown in Figure S-2.

\section{Shotgun Proteomics Analysis of the Mitochondrial} Proteome

The shotgun analysis was performed on the proteins extracted from mitochondrial fractions obtained by two different enrichment protocols on each cell line. All three enrichment protocols were employed on the reference SH-SYSY cells only. The complete

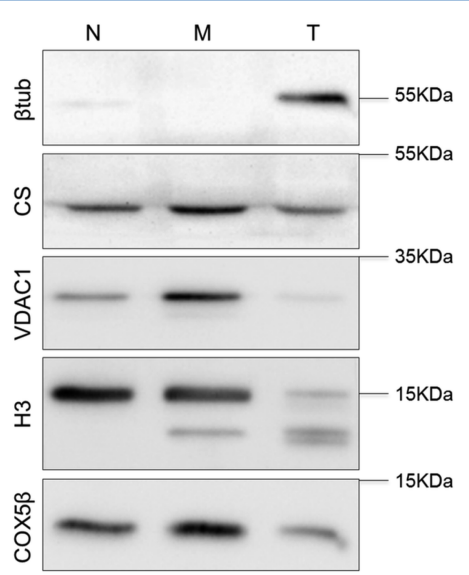

Diff. Centr.

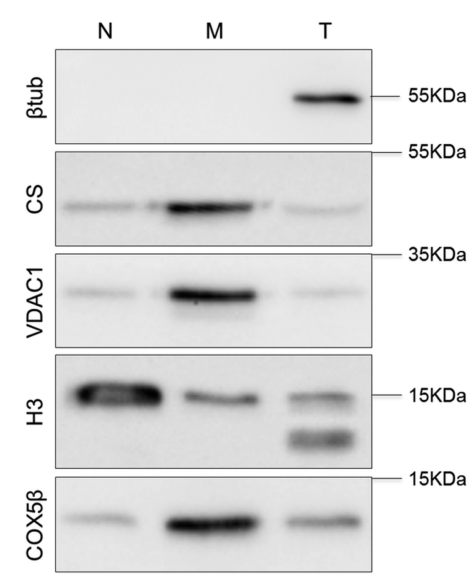

Kit

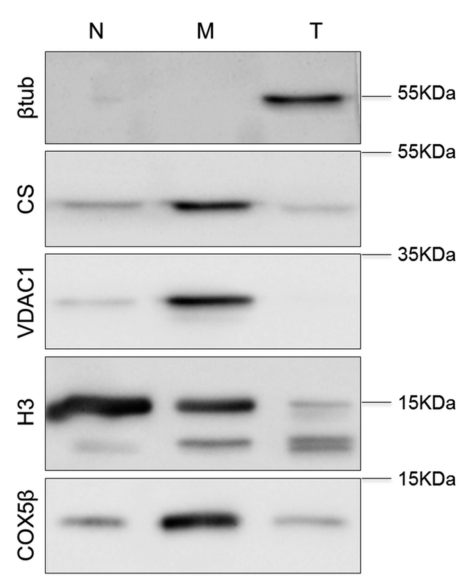

Gradient

Figure 1. Mitochondrial samples yield and purity check. SH-SY5Y mitochondrial enriched fraction obtained by differential centrifugation, the commercial kit based on surfactants and the sucrose gradient protocol. $N=$ nuclear fraction; $M=$ mitochondrial fraction; $T=$ total fraction. 
study design is presented in Figure 2A. This study was planned to ensure a statistically significant number of enrichment replicates for each method and, at the same time, a sufficient randomization so that the origin of the samples and the laboratory performing the isolation were independent. Moreover, to map the large heterogeneity of different LC-MS/MS platforms within the proteomics community, we employed seven different nanoanalytical MS setup, using the same platform for preparations from the same cell line.

Shotgun data from all the laboratories of the Consortium were processed using PEAKS Studio 7.5 as described in the Experimental Procedures section. The raw data were deposited to the ProteomeXchange Consortium via MassIVE repository with the data set identifiers PXD007053 and MSV000081329
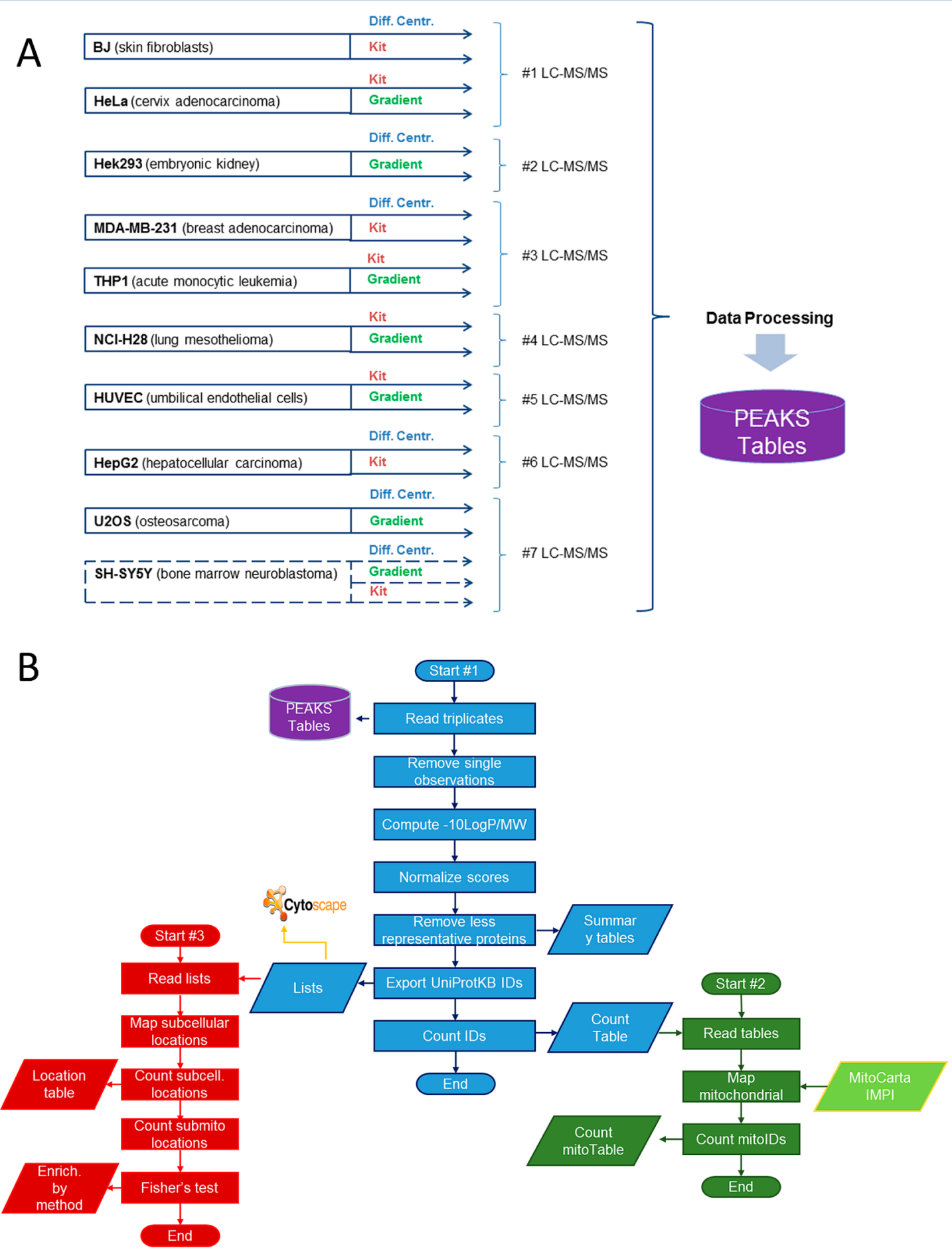

Figure 2. Study design of the mt-HPP consortium standardization action. (A) Workflow from left to right describes the steps of the project in chronological order: the preparation of cell lines with standard procedures, the mitochondrial enrichment with the methods reported, the LC-MS/MS analysis (for details, see Table 1), and the final data analysis. (B) Flowchart describes the three independent routines for the data analysis: the first one (blue) reads the 63 data tables and parses cell lines, mitochondrial enrichment methods, significance scores, Uniprot IDs, and gene symbols; the second one (green) reads the summary tables and maps the IDs observed in each biological replicate against MitoCarta2.0 and IMPI; the third (red) reads the consensus lists and maps their subcellular and submitochondrial locations (codes are reported in the Supporting Information). 
and are freely available for download. The 63 data sets were analyzed using the automated procedure described in Figure 2B and enclosed in the Supporting Information. Briefly, protein lists were filtered by eliminating proteins that were observed in a single preparation and thresholded by retaining all protein identifications whose ranking coefficient $(-10 \log \mathrm{p} / \mathrm{MW})$ was higher than a threshold value equal to $5.0 \times 10^{-4}$ with respect to the sum of all coefficients of the identified proteins in a single experiment. Considering this threshold, the number of identified proteins varied between 200 and 800 , depending on the cell line, the enrichment procedure used, and, in part, the LC-MS/MS technical setup. For some cell lines (NCI-H28, HUVEC, MDAMB-231, THP1), the number of protein identities was significantly dependent on the enrichment procedure (Figure 3A). $\mathrm{SH}-\mathrm{SY} 5 Y$ cells were used as reference model to compare the three mitochondrial enrichment protocols by shotgun analysis (Figure 3B).

The automated procedure allowed the annotation of protein lists using MitoCarta2.0 and IMPI Q2 2016. Protein IDs were considered as mitochondrial if defined as such in at least one of the two databases. On average, the fraction of proteins identified as mitochondrial resulted similar for the three methods (differential centrifugation: $46 \% \pm 11 \%$; sucrose gradient $54 \% \pm 10 \%$; kit based on surfactants $52 \% \pm 11$ ), although differences at the single cell line level were observed (Figure 3C,D).
Evaluation of the Performance of the Mitochondrial Enrichment Protocols

To evaluate the performances of the mitochondrial enrichment procedures, protein IDs were classified using the UniProt "primary subcellular location" category (by grouping information as reported in the Supporting Information). Then data were sorted by the enrichment method. The topmost represented categories for all the methods were "Mitochondrion", "Cytoplasm", "Nucleus", and "ER", respectively, as reported in Figure 4A. In general, the percentage of mitochondrial proteins is not statistically different among the methods, whereas the enrichment of cytoplasmic and nuclear proteins appears significantly different for the three protocols herein considered.

The proteins annotated as mitochondrial were further analyzed to compare their submitochondrial localization in the OMM, the IMS, the IMM, and the mitochondrial Matrix. The results reported in Figure $4 \mathrm{~B}$ indicate that there is no significant difference among the enrichment methods.

In our data sets, the percentages of mitochondrial proteins in each compartment were 9.0\% OMM, 5.2\% IMS, 40.5\% IMM, and $18.5 \%$ Matrix. These numbers are comparable to what was obtained from the same analysis performed on the neXtProt database (9.4\% OMM, 4.9\% IMS, 29\% IMM, and 15.7\% Matrix), with the exception of a higher representation of IMM proteins in our samples. However, the Fisher's exact test revealed no significant difference between our percentages and those expected from
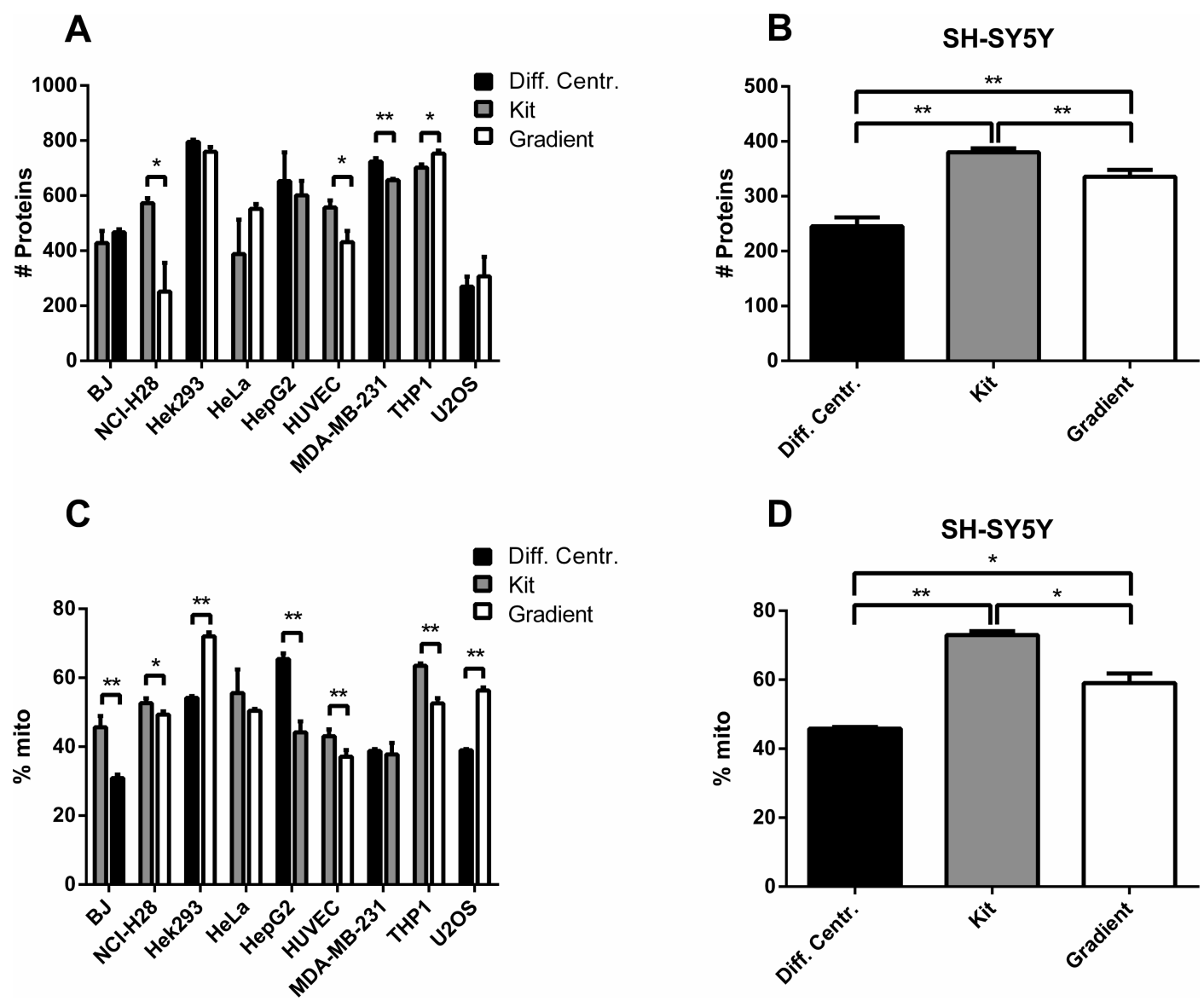

Figure 3. Number of total and mitochondrial proteins identified for each cell line. (A) Total number of proteins identified for each cell line/enrichment method pair (after thresholding as explained in the text). (B) Total number of proteins identified for each of the three mitochondria enrichment method in SH-SY5Y cells (after thresholding as explained in the text). (C) Proportion of mitochondrial proteins for each cell line/enrichment method pair. (D) Percentage of mitochondrial proteins identified for each of the three mitochondria enrichment method in SH-SY5Y cells. Proteins were considered as "mitochondrial" if classified as such in either MitoCarta2.0 or IMPI. Bars represent mean \pm SEM; $* p<0.05, * * p<0.001$. 
A

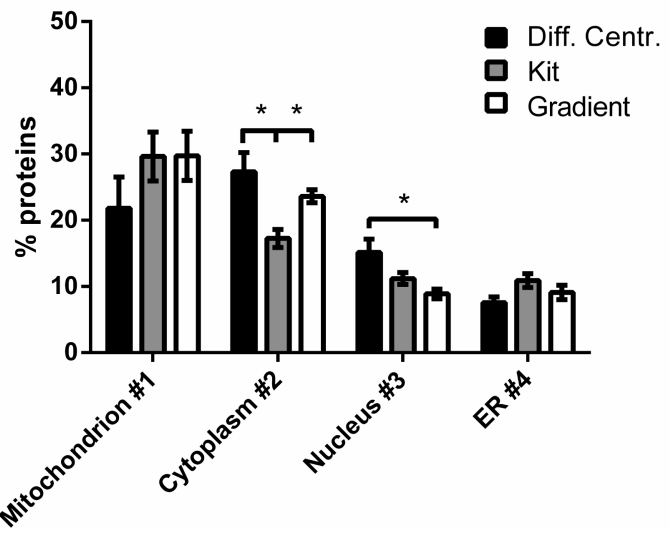

B

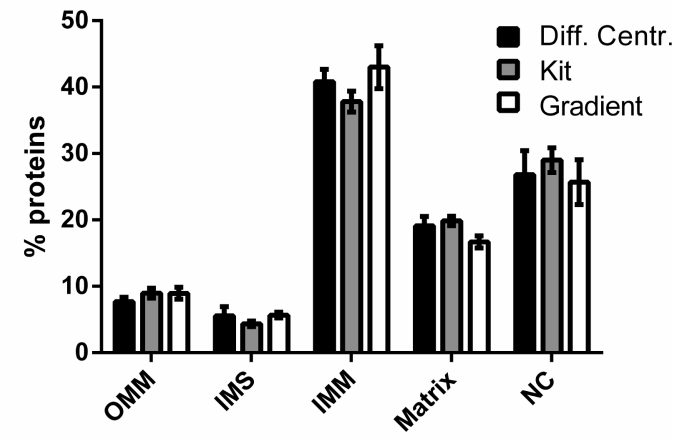

Figure 4. Percentage of four top subcellular locations for each mitochondrial enrichment protocol. (A) Proportion of mitochondrial, cytoplasmic, nuclear, and endoplasmic proteins for each enrichment method (UniProt primary subcellular location). Statistical significance was evaluated with the Kruskal-Wallis test, followed by Dunn's posthoc test. $* p<0.05$. (B) Submitochondrial location, expressed as a percentage of mitochondrial proteins in the OMM, the IMS, on the IMM, or in the mitochondrial Matrix. NC: not classified. Bars represent mean \pm SEM.

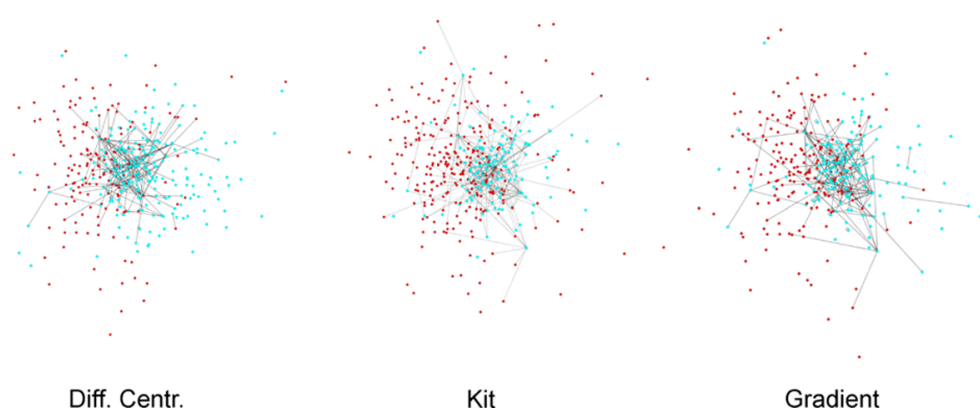

Figure 5. Mapping of the proteins identified from SH-SY5Y cells on the functional mitochondrial reference network. Protein IDs (observed in at least two out of three biological replicates and with a $\left.(-10 \log \mathrm{p} / \mathrm{MW})>5.0 \times 10^{-4}\right)$ were mapped on the reference network of the functional mitochondrial proteome. Cyan nodes represent mitochondrial proteins as annotated in neXtProt, whereas red nodes are their first interactors. ${ }^{10}$ Proportion of IDs are reported in Table 2.

the database. Therefore, we can state that the three methods do not under- or over-represent submitochondrial components.

\section{Mapping on the Reference Network}

The proteins observed in at least two out of three biological replicates for each preparation were mapped on the reference network of the functional mitochondrial proteome. ${ }^{10}$ This procedure allowed us to identify together with the mitochondrial proteins also the proteins that are physically or functionally associated with mitochondria, thus highlighting the ability of each enrichment method to obtain different amounts of proteins that are not strictly mitochondrial, but related to this organelle. Figure 5 reports the mapped proteins for the three protocols on the SH-SY5Y cells. Maps for all the other preparations are reported in the Supporting Information.

Maps were analyzed to extract the total number of mapped proteins, the number of mitochondrial proteins (neXtProt subcellular location) among them, the number of proteins mapping to the largest cluster, and the number of mitochondrial proteins (neXtProt subcellular location) in the cluster. Results are shown in terms of percentage in Table 2.

\section{DISCUSSION}

Mitochondrial dysfunction is widely implicated in the origin and development of various age-associated diseases including metabolic syndromes, cardiovascular diseases, cancer, and neurodegenerative disorders. Bioenergetics aspects linked to mitochondria activity in human disease have provided new opportunities for diagnosis, therapy, prevention, and in connecting various domains of medicine. ${ }^{18}$ Dysfunctions can be assessed in isolated mitochondria. Assays of the amounts or activities of specific complexes and enzymes, mitochondrial morphology, and responses to specific stressors can be useful to test specific hypotheses, but should generally be held in reserve and not used as the primary assay for mitochondrial dysfunction. A more indirect indication of mitochondrial dysfunction is raised by the pattern of changes in gene arrays, proteomics, or metabolomics, where gene ontology categories may suggest alterations in energy metabolism or in other cellular functions where mitochondria play a pivotal role (e.g., apoptosis, calcium homeostasis).

Over the past 20 years, interest in the field of mitochondria proteomics research has increased dramatically. The experimental strategy for defining the mitochondrial proteome is delineated by the application of mass spectrometry to identify proteins from highly enriched mitochondrial extracts. With the improvement in mitochondria purification strategies, together with MS-based proteomics with higher sensitivity and mass accuracy, mitochondrial profiling could represent a powerful technique in studying disease states. However, methods to assess the potential impact of mitochondria purification protocols have been limited. Moreover, mitochondrial proteome in any cell line is delineated by a combination of nuclear chromosomes and mitochondrial 
Table 2. Mapping on the Functional Mitochondrial Proteome ${ }^{a}$

\begin{tabular}{|c|c|c|c|c|c|}
\hline cell line & method & $\%$ mapped & $\%$ mitochondrial & $\%$ clustered & $\%$ mitoclustered \\
\hline \multirow[t]{2}{*}{ BJ } & kit & 77 & 36 & 22 & 53 \\
\hline & diff. centr. & 84 & 21 & 30 & 43 \\
\hline \multirow[t]{2}{*}{ NCI-H28 } & kit & 84 & 46 & 29 & 56 \\
\hline & gradient & 88 & 38 & 28 & 65 \\
\hline \multirow[t]{2}{*}{ Hek293 } & diff. centr. & 87 & 44 & 41 & 58 \\
\hline & gradient & 84 & 63 & 38 & 70 \\
\hline \multirow[t]{2}{*}{ HeLa } & kit & 82 & 46 & 29 & 70 \\
\hline & gradient & 86 & 41 & 34 & 56 \\
\hline \multirow[t]{2}{*}{ HepG2 } & diff. centr & 81 & 57 & 32 & 69 \\
\hline & kit & 78 & 38 & 25 & 65 \\
\hline \multirow[t]{2}{*}{ HUVEC } & kit & 79 & 38 & 33 & 56 \\
\hline & gradient & 82 & 30 & 37 & 49 \\
\hline \multirow[t]{2}{*}{ MDA-MB-231 } & diff. centr. & 79 & 32 & 38 & 49 \\
\hline & kit & 76 & 36 & 36 & 53 \\
\hline \multirow[t]{2}{*}{ THP1 } & kit & 76 & 63 & 36 & 72 \\
\hline & gradient & 78 & 51 & 38 & 62 \\
\hline \multirow[t]{2}{*}{$\mathrm{U} 2 \mathrm{OS}$} & diff. centr. & 83 & 30 & 29 & 53 \\
\hline & gradient & 87 & 45 & 30 & 66 \\
\hline \multirow[t]{3}{*}{ SH-SY5Y } & diff. centr. & 89 & 33 & 28 & 54 \\
\hline & kit & 90 & 60 & 26 & 66 \\
\hline & gradient & 89 & 47 & 32 & 64 \\
\hline
\end{tabular}

${ }^{a}$ Notes: diff. centr, differential centrifugation; gradient, sucrose gradient; \% mapped, percentage of protein IDs mapped on the reference network (mapped/total IDs); \% mitochondrial, percentage of mapped IDs annotated as mitochondrial in neXtProt (mitochondrial/mapped); \% clustered, percentage of mapped IDs that cluster in the main subnetwork (clustered/mapped); \% mitoclustered, percentage of clustered IDs annotated as mitochondrial in neXtProt (mitochondrial/clustered).

chromosome-coded proteins, together with their interacting structures. Given these requirements and constraints, it is hardly surprising that a relatively small number of largely complete and robustly validated mitochondrial proteomes were reported so far.

In this work, we assessed the performances of three different purification methods on ten human cell lines of different origin. The human cell lines were selected because they are some of the most thoroughly characterized cell lines in biomedical research ${ }^{9}$ (i.e., HeLa, Hek293, U2OS) or because they are commonly used cellular models for specific conditions. Many of them are included in the panel used for the mapping of Cell Atlas subcellular locations within the Human Protein Atlas project. ${ }^{9}$ Moreover, they include normal noncancer cells such as umbilical endothelial cells (HUVEC) and skin fibroblasts (BJ). BJ human fibroblast cells were one of the first cell types to be reprogrammed into induced pluripotent stem (iPS) cells and are commonly used in human cell reprogramming studies. ${ }^{19}$ Moreover, the use of fibroblasts is emerging in different research fields. Since they are relatively easy to obtain from patients skin biopsy, they provide a unique cell system for personalized medicine. ${ }^{20-22}$ SH-SY5Y are widely used in neuroscience research as a neuron-like model easy to grow and maintain indefinitely. ${ }^{23-27}$ MDA-MB-231 are one of the most frequently used cell lines for in vitro experimental study of the hormone-independent breast cancer. The mitochondrial respiration defects observed in MDA-MB-231 cells were demonstrated to be caused by mutations in mtDNA, which in turn is responsible for the high metastatic potential. ${ }^{28-30}$ NCI-H28 is a malignant mesothelioma cell line, normally used to investigate this pathological condition. ${ }^{31}$ Endothelial cells (ECs), such as HUVEC, are suitable for the study of endothelial physiology, vascular functions, and interaction of ECs with blood cells and various mediators, all of which are relevant in different diseases, such as atherosclerosis, inflammation, and immunity. ${ }^{32}$ THP1 is a human leukemia monocytic cell line, which can be easily differentiated into a macrophage phenotype, and hence, it has been extensively used to study monocyte/macrophage functions. ${ }^{33,34}$ HepG 2 cell line, which was derived from a liver hepatocellular carcinoma, is a good cellular model to isolate mitochondria, to perform measurements of mitochondrial respiration, to identify drug-induced mitochondrial toxicity, and to evaluate the role of mitochondrial dysfunction in cancer progression. ${ }^{35-37}$ Far to be representative of all the cellular models used in mitochondrial research, our cell panel should be considered as a starting working hypothesis to improve mitochondrial enrichment for proteomics studies.

The purification methods consisted of an isolation kit based on a surfactant cell lysis, differential centrifugation, and sucrose gradient separation, based on mechanical homogenization in a nonionic osmotic solution, where sugars are used as osmolytes. First, we assessed purity and yield of the enriched fractions by Western blot. Nevertheless, this simple assay may not be sufficient to validate each fraction undergoing LC-MS/MS. Actually, long mitochondrial chains may break out during isolation, thus leading to loss of Matrix proteins. Similarly, the mechanical stress during the extraction procedure may improve the formation of mitoplasts, where OMM and IMS proteins are underrepresented. Therefore, we assessed mitochondria integrity by measuring the citrate synthase activity and the oxygen consumption rate of enriched mitochondria in the most problematic samples. The results indicated that mitochondrial integrity is retained and it is independent of yield and purity. Moreover, we verified a posteriori the submitochondrial location of mitochondrial proteins according to UniProt annotation. Their relative proportion is in agreement with that of the neXtProt database, revealing that the proteomics analysis was performed on intact mitochondria.

Three biological replicates for each mitochondria enrichment protocol were prepared and analyzed separately in technical duplicate by shotgun proteomics. This is a strong constrain 
to ensure that shotgun data are biologically representative and meaningful. ${ }^{38}$ Indeed, the observation of a single pooled sample, in the absence of biological replicates, may lead to neglecting the intrinsic variability of specimens. For this reason, only proteins observed in at least two out of the three biological replicates were taken into account.

The study design was developed to highlight differences in the enrichment protocols, which are independent of the analytical set up of the LC-MS/MS systems. Thus, we employed a variety of different LC-MS/MS platforms, however maintaining the same set up within the same cell line. Another important issue to be faced is the number of identifications that different chromatographic systems coupled to different mass spectrometers may provide. It is straightforward that lists of different lengths representing similar samples will share the identities of most abundant proteins. We tackled this issue by analyzing samples isolated with different methods from the same cell line using the same instrument. Additionally, we ranked identified proteins in terms of the significance score over the molecular weight, a concept close to the Exponentially Modified Protein Abundance Index (emPAI). ${ }^{14}$ Significance $(-10 \log p)$ scores reflect peptide-spectrum and protein-peptide matches and the molecular weight reflects the length of the protein sequence. After thresholding (i.e., retaining proteins with the rank score higher than a threshold), the dimension of the lists was independent of the instrumental platform. Noticeably, the thresholding procedure was necessary only for comparison purposes of the present work and does not strictly reflect a label-free quantification of the proteins. Analysis of the thresholded data revealed no significant differences in terms of number of identified proteins among the three methods. Although with the limitation represented by the use of two preparative methods in the other cell lines, significant discrepancies were observed for specific cells. In particular, the differential centrifugation protocol was better suited for MDA-MB-231, the sucrose gradient seemed to work better than the kit for THP1 cells, whereas the number of identified proteins was higher with the kit for NCI-H28, HUVEC, and SH-SY5Y cells.

A first approach to determine the performance of the enrichment protocols in terms of mitochondrial proteins identified in each experiment relied on reference databases such as MitoCarta2.0 and IMPI Q2 2016. Merging the results for each enrichment protocol, mitochondrial proteins always accounted for about $50 \%$ and, consequently, differences associated with the enrichment method were not significant. However, a significantly higher enrichment was observed for HepG2 cells, where differential centrifugation recovers more mitochondrial proteins than the kit, and for Hek293 and U2OS cells, where sucrose gradient works better than differential centrifugation. For BJ, NCI-H28, HUVEC, THP1, and SH-SY5Y cells, the kit gives the best results. Therefore, these findings should guide researchers through the choice of the appropriate method for a given cell line. A second analysis to evaluate the specificity of the enrichment procedures was performed considering the primary subcellular location as annotated in UniProt. This method is not sensitive, because mitochondrial proteins may have a location other than mitochondrial as the primary annotation. However, one can retrieve a general information about the occurrence of the four most frequent primary locations. Results show that the kit allows for a significantly lower enrichment in cytoplasmic proteins, whereas the gradient leads to the lower contamination by nuclear proteins.

One of the key points of the present study is the ability to selectively coisolate other cell structures associated with mitochondria. To this purpose, we cannot rely on "classical" databases. Recently, we proposed the concept of the functional mitochondrial proteome as a large (6592 nodes) network encompassing all the proteins labeled as mitochondrial in neXtProt together with their first interactors (IMEX consortium, 5231 nodes). Noticeably, mapping the protein lists on this network allowed us to match more of $80 \%$ identities, thus showing that nonmitochondrial proteins are indeed associated with mitochondria, from a functional point of view, and can be enriched together with mitochondria. If we again consider SH-SY5Y cells to compare three methods, we observe that $89 \pm 1 \%$ identities are mapped on the reference network, whereas a variable percentage of them is strictly mitochondrial, ranging from $33 \%$ for the differential centrifugation protocol to $60 \%$ for the kit. Thus, relative data in Table 2 should provide a background for the choice of the preferred method, privileging purity of enriched mitochondria or coenriching associated structures. Further elements may arise from the analysis of the size of the main cluster and of mitochondrial proteins within, showing the ability to recover entire supramolecular complexes. This is particularly important for proteins that dynamically interact with mitochondria and exert a chronosteric effect (e.g., parkin, which was never identified in the present study).

In conclusion, we have compared suitable procedures to achieve an effective MS analysis of the mitochondrial proteome, defining the most advisible enrichment method for different experimental designs, irrespective of the analytical LC-MS/MS platform employed. This standardization action will advisibly contribute to future projects of the mt-HPP of the B/D-HPP and add several mitochondrial data sets on ProteomeXchange to be used as reference for future proteomics studies. We do not draw a unique conclusion, as no method is preferable as such. We instead provide some interesting hints for the different cellular models used. From a biological perspective, the investigation of the mitochondrial proteome from an integrated, functional point of view should improve the comprehensive vision of key signaling pathways and biological processes converging onto the mitochondrion. Convergent signals are sensitive to the purification methods used, offering the opportunity for increasing the knowledge of biochemical and genetic aspects of mitochondrial biology and pathology. Of particular interest will be the elucidation of mitochondria-nuclear crosstalks that regulate cell-specific features, ${ }^{39}$ which are themselves epigenetically determined. ${ }^{40}$

\section{ASSOCIATED CONTENT}

\section{Supporting Information}

The Supporting Information is available free of charge on the ACS Publications website at DOI: 10.1021/acs.jproteome.7b00350.

Cell culture conditions; R analysis of MS data; Western blots; functional characterization; detailed procedures for LC-MS/MS; automated procedure for analysis of shotgun results; cell culture and cell lysis conditions; verification of mitochondrial samples yield and purity; functional characterization of mitochondrial isolations; mapping of protein lists on functional mitochondrial reference network (PDF)

\section{AUTHOR INFORMATION}

\section{Corresponding Author}

*E-mail: mauro.fasano@uninsubria.it. 


\section{ORCID 1}

Mauro Fasano: 0000-0003-0628-5871

Author Contributions

$\Phi_{\text {Equal contribution. }}$

Notes

The authors declare no competing financial interest.

The raw data were deposited to the ProteomeXchange Consortium via MassIVE repository with the data set identifiers PXD007053 and MSV000081329 and are freely available for download.

\section{ACKNOWLEDGMENTS}

We acknowledge Emma Lundberg for conveying us the BJ (ATCC CRL-2522) and U2OS (ATCC HTB-96) cell lines, and all the co-workers in the laboratories of the mt-HPP consortium for valuable help in all the tasks of the present project.

\section{REFERENCES}

(1) Urbani, A.; De Canio, M.; Palmieri, F.; Sechi, S.; Bini, L.; Castagnola, M.; Fasano, M.; Modesti, A.; Roncada, P.; Timperio, A. M.; Bonizzi, L.; Brunori, M.; Cutruzzolà, F.; De Pinto, V.; Di Ilio, C.; Federici, G.; Folli, F.; Foti, S.; Gelfi, C.; Lauro, D.; Lucacchini, A.; Magni, F.; Messana, I.; Pandolfi, P. P.; Papa, S.; Pucci, P.; Sacchetta, P.; Italian Mt-Hpp Study Group-Italian Proteomics Association. The mitochondrial Italian Human Proteome Project initiative (mt-HPP). Mol. Biosyst. 2013, 9, 1984-1992.

(2) Kasahara, A.; Scorrano, L. Mitochondria: from cell death executioners to regulators of cell differentiation. Trends Cell Biol. 2014, 24, 761-770.

(3) Modica-Napolitano, J. S.; Singh, K. K. Mitochondrial dysfunction in cancer. Mitochondrion 2004, 4, 755-762.

(4) Lowell, B. B.; Shulman, G. I. Mitochondrial dysfunction and type 2 diabetes. Science 2005, 307, 384-387.

(5) Lin, M. T.; Beal, M. F. Mitochondrial dysfunction and oxidative stress in neurodegenerative diseases. Nature 2006, 443, 787-795.

(6) Rafelski, S. M. Mitochondrial network morphology: building an integrative, geometrical view. BMC Biol. 2013, 11, 71.

(7) Hoppins, S. The regulation of mitochondrial dynamics. Curr. Opin. Cell Biol. 2014, 29, 46-52.

(8) Yin, F.; Cadenas, E. Mitochondria: the cellular hub of the dynamic coordinated network. Antioxid. Redox Signaling 2015, 22, 961-964.

(9) Thul, P. J.; Åkesson, L.; Wiking, M.; Mahdessian, D.; Geladaki, A.; Ait Blal, H.; Alm, T.; Asplund, A.; Björk, L.; Breckels, L. M.; Bäckström, A.; Danielsson, F.; Fagerberg, L.; Fall, J.; Gatto, L.; Gnann, C.; Hober, S.; Hjelmare, M.; Johansson, F.; Lee, S.; Lindskog, C.; Mulder, J.; Mulvey, C. M.; Nilsson, P.; Oksvold, P.; Rockberg, J.; Schutten, R.; Schwenk, J. M.; Sivertsson, Å.; Sjöstedt, E.; Skogs, M.; Stadler, C.; Sullivan, D. P.; Tegel, H.; Winsnes, C.; Zhang, C.; Zwahlen, M.; Mardinoglu, A.; Pontén, F.; von Feilitzen, K.; Lilley, K. S.; Uhlén, M.; Lundberg, E. A subcellular map of the human proteome. Science 2017, 356, eaal3321.

(10) Fasano, M.; Alberio, T.; Babu, M.; Lundberg, E.; Urbani, A. Towards a functional definition of the mitochondrial human proteome. EuPa Open Proteomics 2016, 10, 24-27.

(11) R Core Team. R: A Language and Environment for Statistical Computing; R Foundation for Statistical Computing: Vienna, Austria, 2014. http://www.R-project.org/.

(12) Carlson, M. UniProt.ws: R Interface to UniProt Web Services. $R$ Package Version 2.14.0; R Foundation for Statistical Computing, 2016.

(13) Huber, W.; Carey, V. J.; Gentleman, R.; Anders, S.; Carlson, M.; Carvalho, B. S.; Bravo, H. C.; Davis, S.; Gatto, L.; Girke, T.; Gottardo, R.; Hahne, F.; Hansen, K. D.; Irizarry, R. A.; Lawrence, M.; Love, M. I.; MacDonald, J.; Obenchain, V.; Oleś, A. K.; Pagès, H.; Reyes, A.; Shannon, P.; Smyth, G. K.; Tenenbaum, D.; Waldron, L.; Morgan, M. Orchestrating high-throughput genomic analysis with Bioconductor. Nat. Methods 2015, 12, 115-121.
(14) Ishihama, Y.; Oda, Y.; Tabata, T.; Sato, T.; Nagasu, T.; Rappsilber, J.; Mann, M. Exponentially modified protein abundance index (emPAI) for estimation of absolute protein amount in proteomics by the number of sequenced peptides per protein. Mol. Cell. Proteomics 2005, 4, 12651272.

(15) Calvo, S. E.; Clauser, K. R.; Mootha, V. K. MitoCarta2.0: an updated inventory of mammalian mitochondrial proteins. Nucleic Acids Res. 2016, 44, D1251-1257.

(16) Smith, A. C.; Robinson, A. J. MitoMiner v3.1, an update on the mitochondrial proteomics database. Nucleic Acids Res. 2016, 44, D1258-1261.

(17) Shannon, P.; Markiel, A.; Ozier, O.; Baliga, N. S.; Wang, J. T.; Ramage, D.; Amin, N.; Schwikowski, B.; Ideker, T. Cytoscape: a software environment for integrated models of biomolecular interaction networks. Genome Res. 2003, 13, 2498-2504.

(18) Picard, M.; Wallace, D. C.; Burelle, Y. The rise of mitochondria in medicine. Mitochondrion 2016, 30, 105-116.

(19) Yu, J.; Vodyanik, M. A.; Smuga-Otto, K.; Antosiewicz-Bourget, J.; Frane, J. L.; Tian, S.; Nie, J.; Jonsdottir, G. A.; Ruotti, V.; Stewart, R.; Slukvin, I. I.; Thomson, J. A. Induced pluripotent stem cell lines derived from human somatic cells. Science 2007, 318, 1917-1920.

(20) Auburger, G.; Klinkenberg, M.; Drost, J.; Marcus, K.; MoralesGordo, B.; Kunz, W. S.; Brandt, U.; Broccoli, V.; Reichmann, H.; Gispert, S.; Jendrach, M. Primary skin fibroblasts as a model of Parkinson's disease. Mol. Neurobiol. 2012, 46, 20-27.

(21) Qiang, L.; Inoue, K.; Abeliovich, A. Instant neurons: directed somatic cell reprogramming models of central nervous system disorders. Biol. Psychiatry 2014, 75, 945-951.

(22) Tigges, J.; Krutmann, J.; Fritsche, E.; Haendeler, J.; Schaal, H.; Fischer, J. W.; Kalfalah, F.; Reinke, H.; Reifenberger, G.; Stühler, K.; Ventura, N.; Gundermann, S.; Boukamp, P.; Boege, F. The hallmarks of fibroblast ageing. Mech. Ageing Dev. 2014, 138, 26-44.

(23) Kovalevich, J.; Langford, D. Considerations for the use of SHSY5Y neuroblastoma cells in neurobiology. Methods Mol. Biol. 2013, 1078, 9-21.

(24) Alberio, T.; Lopiano, L.; Fasano, M. Cellular models to investigate biochemical pathways in Parkinson's disease. FEBS J. 2012, 279, $1146-$ 1155.

(25) Alberio, T.; Bondi, H.; Colombo, F.; Alloggio, I.; Pieroni, L.; Urbani, A.; Fasano, M. Mitochondrial proteomics investigation of a cellular model of impaired dopamine homeostasis, an early step in Parkinson's disease pathogenesis. Mol. BioSyst. 2014, 10, 1332-1344.

(26) Krishna, A.; Biryukov, M.; Trefois, C.; Antony, P. M.; Hussong, R.; Lin, J.; Heinäniemi, M.; Glusman, G.; Köglsberger, S.; Boyd, O.; van den Berg, B. H.; Linke, D.; Huang, D.; Wang, K.; Hood, L.; Tholey, A.; Schneider, R.; Galas, D. J.; Balling, R.; May, P. Systems genomics evaluation of the SH-SY5Y neuroblastoma cell line as a model for Parkinson's disease. BMC Genomics 2014, 15, 1154.

(27) Xicoy, H.; Wieringa, B.; Martens, G. J. M. The SH-SY5Y cell line in Parkinsonís disease research: a systematic review. Mol. Neurodegener. 2017, 12, 10.

(28) Tseng, L. M.; Yin, P. H.; Yang, C. W.; Tsai, Y. F.; Hsu, C. Y.; Chi, C. W.; Lee, H. C. Somatic mutations of the mitochondrial genome in human breast cancers. Genes, Chromosomes Cancer 2011, 50, 800-811.

(29) Imanishi, H.; Hattori, K.; Wada, R.; Ishikawa, K.; Fukuda, S.; Takenaga, K.; Nakada, K.; Hayashi, J. Mitochondrial DNA mutations regulate metastasis of human breast cancer cells. PLoS One 2011, 6, e23401.

(30) Tu, Y. F.; Kaipparettu, B. A.; Ma, Y.; Wong, L. J. Mitochondria of highly metastatic breast cancer cell line MDA-MB-231 exhibits increased autophagic properties. Biochim. Biophys. Acta, Bioenerg. 2011, 1807, 1125-1132.

(31) Fazzini, A.; D’Antongiovanni, V.; Giusti, L.; Da Valle, Y.; Ciregia, F.; Piano, I.; Caputo, A.; D’Ursi, A. M.; Gargini, C.; Lucacchini, A.; Mazzoni, M. R. Altered protease-activated receptor-1 expression and signaling in a malignant pleural mesothelioma cell line, NCI-H28, with homozygous deletion of the $\beta$-catenin gene. PLoS One 2014, 9, e111550.

(32) Onat, D.; Brillon, D.; Colombo, P. C.; Schmidt, A. M. Human vascular endothelial cells: a model system for studying vascular 
inflammation in diabetes and atherosclerosis. Curr. Diabetes Rep. 2011, 11, 193-202.

(33) Qin, Z. The use of THP-1 cells as a model for mimicking the function and regulation of monocytes and macrophages in the vasculature. Atherosclerosis 2012, 221, 2-11.

(34) Chanput, W.; Mes, J. J.; Wichers, H. J. THP-1 cell line: an in vitro cell model for immune modulation approach. Int. Immunopharmacol. 2014, 23, 37-45.

(35) Yin, P. H.; Wu, C. C.; Lin, J. C.; Chi, C. W.; Wei, Y. H.; Lee, H. C. Somatic mutations of mitochondrial genome in hepatocellular carcinoma. Mitochondrion 2010, 10, 174-182.

(36) Kamalian, L.; Chadwick, A. E.; Bayliss, M.; French, N. S.; Monshouwer, M.; Snoeys, J.; Park, B. K. The utility of HepG2 cells to identify direct mitochondrial dysfunction in the absence of cell death. Toxicol. In Vitro 2015, 29, 732-740.

(37) Costantini, S.; Di Bernardo, G.; Cammarota, M.; Castello, G.; Colonna, G. Gene expression signature of human HepG2 cell line. Gene 2013, 518, 335-345.

(38) Yates, J. R., III. Change. J. Proteome Res. 2016, 15, 2355.

(39) Hämäläinen, R. H.; Manninen, T.; Koivumäki, H.; Kislin, M.; Otonkoski, T.; Suomalainen, A. Tissue- and cell-type-specific manifestations of heteroplasmic mtDNA 3243A > G mutation in human induced pluripotent stem cell-derived disease model. Proc. Natl. Acad. Sci. U. S. A. 2013, 110, E3622-3630.

(40) Meissner, A.; Mikkelsen, T. S.; Gu, H.; Wernig, M.; Hanna, J.; Sivachenko, A.; Zhang, X.; Bernstein, B. E.; Nusbaum, C.; Jaffe, D. B.; Gnirke, A.; Jaenisch, R; Lander, E. S. Genome-scale DNA methylation maps of pluripotent and differentiated cells. Nature 2008, 454, 766-770. 